\title{
Connecting Moving Groups to the Bar and Spiral Arms of the Milky Way
}

\author{
T. Antoja ${ }^{1}$, O. Valenzuela ${ }^{2}$, F. Figueras ${ }^{1}$, B. Pichardo ${ }^{2}$ \& E. Moreno ${ }^{2}$ \\ ${ }^{1}$ DAM and IEEC-UB, ICC-Universitat de Barcelona, Spain, email: tantoja@am.ub.es \\ ${ }^{2}$ Instituto de Astronomia de la UNAM, Mexico, email: octavio@astroscu.unam.mx
}

\begin{abstract}
We use test-particle orbit integration with a realistic Milky Way (MW) potential to study the effect of the resonances of the Galactic bar and spiral arms on the velocity distribution of the Solar Neighbourhood and other positions of the disk. Our results show that spiral arms create abundant kinematic substructure and crowd stars into the region of the Hercules moving group in the velocity plane. Bar resonances can contribute to the origin of low-angular momentum moving groups like Arcturus. Particles in the predicted dark disk of the MW should be affected by the same resonances as stars, triggering dark-matter moving groups in the disk. Finally, we evaluate how this study will be advanced by upcoming Gaia data.
\end{abstract}

Keywords. Galaxy: disk, kinematics and dynamics, structure, solar neighborhood, dark matter

The MW potential and initial conditions (cold, intermediate and hot disks) of our simulations are described in Antoja et al. (2009). Next figures show: a) left: the region of Hercules $\left(V=-40 \mathrm{~km} \mathrm{~s}^{-1}\right)$ is crowded by the the spiral arms applied to the cold disk, b) middle: the bar with the hot disk creates groups at low angular momentum $\left(V=-100 \mathrm{~km} \mathrm{~s}^{-1}\right)$ with long integration time, c) right: effects of a model with spiral arms and bar. We propose that a good fit between the observed velocity field - see Antoja et al. (2008) - and the simulations requires the combined model under IC1, IC2 and IC3, where the central and low angular momentum moving groups would appear simultaneously.
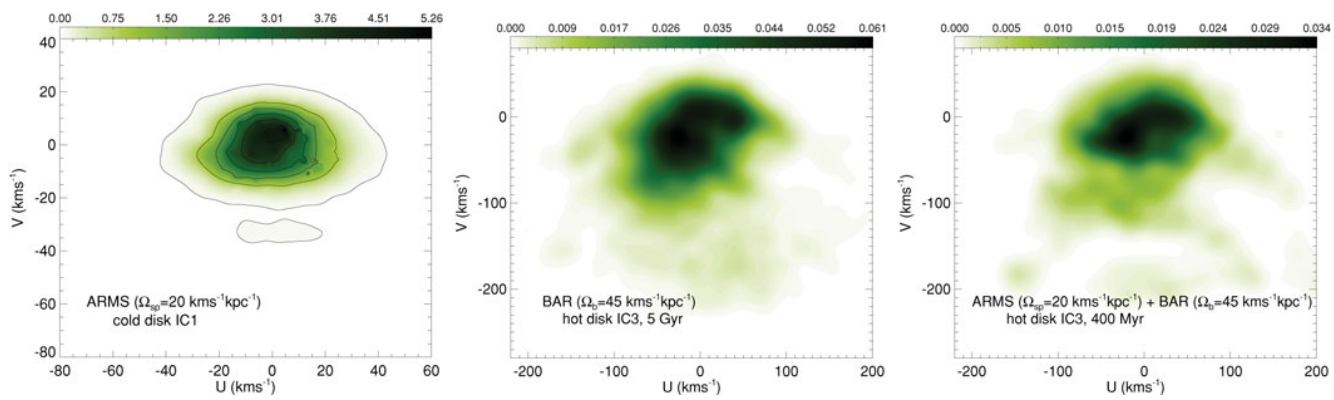

Gaia will revolutionize our knowledge of the Galactic disk. Accuracies in $U V W$ velocities are computed using the Gaia Universe Model Snapshot (GUMS), based on the Besançon Galaxy Model, and the current estimations of the Gaia errors. We find that we will be able to perform robust statistical analysis of the velocity distribution (with accuracies better than $2 \mathrm{~km} \mathrm{~s}^{-1}$ in all components $\left.U V W\right)$ up to $\sim 3 \mathrm{kpc}$ from the Sun.

\section{References}

Antoja, T., Figueras, F., Fernández, F., et al. 2008, A\&A, 490, 135

Antoja, T., Valenzuela, O., Pichardo, B. et al. 2009, ApJL, 700, 78 